\title{
Kinetic theory for strong uniform shear flow of granular media at high density
}

\author{
W. Till Kranz ${ }^{1, \star}$ and Matthias Sperl ${ }^{1,2}$ \\ ${ }^{1}$ Institut für Materialphysik im Weltraum, Deutsches Zentrum für Luft- und Raumfahrt e.V., Linder Höhe, 51147 Köln, Germany \\ ${ }^{2}$ Institut für Theoretische Physik, Universität zu Köln, Zülpicher Straße 77, 50937 Köln, Germany
}

\begin{abstract}
We discuss the uniform shear flow of a fluidized granular bed composed of monodisperse Hertzian spheres. Considering high densities around the glass transition density of inelastic Hertzian spheres, we report kinetic theory expressions for the Newtonian viscosity as well as the Bagnold coefficient. We discuss the dependence of the transport coefficients on density and coefficient of restitution.
\end{abstract}

\section{Introduction}

Flows of dense granular materials are ubiquitous in nature and industry [1-5]. For efficiency [6] and disaster prevention [7] a solid understanding of the rheology of these flows is crucial. Inclined plane flows both in industrial and geophysical settings have been successfully modeled by inelastic Hertzian spheres [5, 8-11] using molecular-dynamics simulations. Discrete element models are constrained, however, to relatively small system sizes due to computational demands.

For a continuum modeling which can be applied at very large scales and a theoretical analysis, it is essential to have access to the transport coefficients as functions of the system parameters. For low densities, the kinetic theory of granular flows is well developed based on the inelastic Boltzmann equation [12-14]. It yields predictions for, amongst others, the diffusivity and the shear viscosity. For high densities and especially away from the linear response regime, results derived from first principles are rare.

The Integration Through Transients (ITT) formalism has been very successful in describing shear in colloidal suspensions at densities around the glass transition and for finite shear rates [15-17]. A glass transition is observed [18] and predicted for fluidized granular beds also [19, 20]. This allowed us recently to generalize the ITT formalism to the far from equilibrium regime of a driven granular medium [21]. In this contribution we will present results for the viscosity and Bagnold coefficient. Hayakawa et al. have derived two related sets of equations (Refs. [22] and [23]) where only the second version has yielded numerical results for the transport coefficients so far.

The structure of the paper is as follows. In Sec. 2 we will define the model and control parameters. A brief sketch of the theory and the required inputs are given in Sec. 3 before we will discuss the transport coefficients for the

\footnotetext{
^e-mail: till.kranz@dlr.de
}

limiting cases of weak (Sec. 4) and strong (Sec. 5) shear. We will close with a discussion in Sec. 6.

\section{Model}

We consider a monodisperse system of $N \rightarrow \infty$ inelastic Hertzian spheres [24, 25] of diameter $d$ and mass $m=1$ in a volume $V$ such that the density $n=N / V$ remains finite. The overlap potential is given by

$$
V\left(r_{i j}\right)=\Gamma T\left(1-r_{i j} / d\right)^{5 / 2} \text { for } r_{i j}<d
$$

where $\Gamma$ controls the strength of the repulsion and $T=$ $\left\langle\boldsymbol{v}^{2}\right\rangle / 3$ is the granular temperature. The distance between two particles is denoted by $r_{i j}=\left|\boldsymbol{r}_{i}-\boldsymbol{r}_{j}\right|$. Here we will focus on the practically relevant case of rather stiff particles, $\Gamma \gg 1$. Dissipation is quantified by a constant coefficient of restitution $0 \leq \epsilon<1$ [12].

We consider the quiescent system to be in a fluidized state $[18,26,27]$ and model the fluidization by a Gaussian random force $\boldsymbol{\xi}_{i}(t)$ with zero mean $\left\langle\boldsymbol{\xi}_{i}\right\rangle=0$ acting on each particle individually. Its variance $\left\langle\xi_{i}^{\alpha}(t) \xi_{j}^{\beta}\left(t^{\prime}\right)\right\rangle=$ $2 P_{D} \delta_{i j} \delta^{\alpha \beta} \delta\left(t-t^{\prime}\right)$ is characterized by the driving power $P_{D}$

We imagine that at time $t=0$ the system is in a stationary state where the dissipation is balanced by the driving force resulting in a stationary temperature $T_{0}$. At this point we switch on a uniform linear shear profile modeled by the SLLOD equations of motion [28]. We prescribe the shear rate $\dot{\gamma}$ and monitor the resulting shear stress $\sigma$. For finite shear rates the shear will contribute to the energy input by a term $\dot{\gamma} \sigma$.

If we wait long enough the system will reach a new stationary state with a new temperature $T$ and constant mean shear stress $\sigma$. This is the state we want to consider in the following. For a given material of the particles prescribed by the pair $(\epsilon, \Gamma)$, the macroscopic state of the system is fully specified by the packing fraction $\varphi=\pi n d^{3} / 6$, the temperature $T_{0}$ (or, equivalently, $T$ ) resulting from the energy balance, and the shear rate $\dot{\gamma}$. 


\section{Calculating the Transport Coefficients}

The transport coefficients which have traditionally been calculated from the Boltzmann-Enskog equation using, e.g., the Chapmann-Enskog or related methods [29], are necessarily valid only to lowest order in the shear rate $\dot{\gamma}$ and the packing fraction $\varphi$. To go beyond the lowest order presents a considerable challenge.

For densities around the (dynamic) glass transition, the ITT formalism enables to calculate corrections beyond the linear order in packing fraction and for arbitrary shear rates. It relates expectation values in the stationary sheared state to the (equilibrium) unsheared state and captures the transient dynamics after the switch-on of shear in terms of time-dependent density correlation functions [17]. For the latter, mode-coupling theory (MCT) [30] affords a faithful description for the densities under consideration.

Granular MCT allows for a generalization of the ITT formalism for dissipative particles. A crucial input for both MCT and ITT are static structure factors, $S_{k}$, in the quiescent state. To this end we use numerical solutions of the Hypernetted-Chain-Equation [31] for Hertzian spheres [32].

For the low-density (bare) transport coefficients, we use the Enskog expressions provided by Garzó and Montanero [14]. Especially important are the shear viscosity $\eta_{0}$ and the related sound-damping constant $D_{S}$.

\section{Linear Rheology}

For small shear rate $\dot{\gamma} \rightarrow 0$ and packing fractions $\varphi<\varphi_{c}$ below the glass transition, we recover the linear response of a Newtonian fluid. We consider two values for the Hertzian stiffness $\Gamma=1000$ and $\Gamma=1500$ as well as several values for the coefficient of restitution $\epsilon$. For the packing fraction we choose values high enough for the validity of our approach but well below the hard-sphere random close packing density $\varphi_{J} \approx 0.64$ [33] such that we avoid lasting contacts between particles.

The ITT formalism yields the following expression for the viscosity

$$
\eta=\eta_{0}+\frac{3}{10 \pi^{2}} \times \frac{(1+\epsilon)^{2}}{4} \times \frac{T}{d D_{S}} \varphi^{2} \chi^{2} \tilde{\eta}[S]\left(c d / D_{S}\right)
$$

where $\chi$ is the pair correlation function at contact, $c$ denotes the (long wavelength) speed of sound, and ${ }^{1}$

$$
\tilde{\eta}[S](K)=\int_{0}^{\infty} d k \frac{K^{2}+2 k^{2}}{K^{2}+k^{2}} \times \frac{\left[j_{0}^{\prime \prime}(k)\right]^{2}}{S_{k}^{2}} .
$$

We expect that generally the viscosity will be lower for more dissipative particles (smaller $\epsilon$ ) but note that $D_{S}$ strongly depends on $\epsilon$ especially for nearly elastic particles [14]. The influence of the particles stiffness $\Gamma$ will enter through the static structure factor $S_{k}$.

For moderate densities (cf. Fig. 1), we observe that the viscosity increases with $\epsilon$ as expected. Note that we obtain values that are far above the Boltzmann-Enskog value

\footnotetext{
${ }^{1}$ Here $j_{0}(x)$ is the zeroth order spherical Bessel function.
}

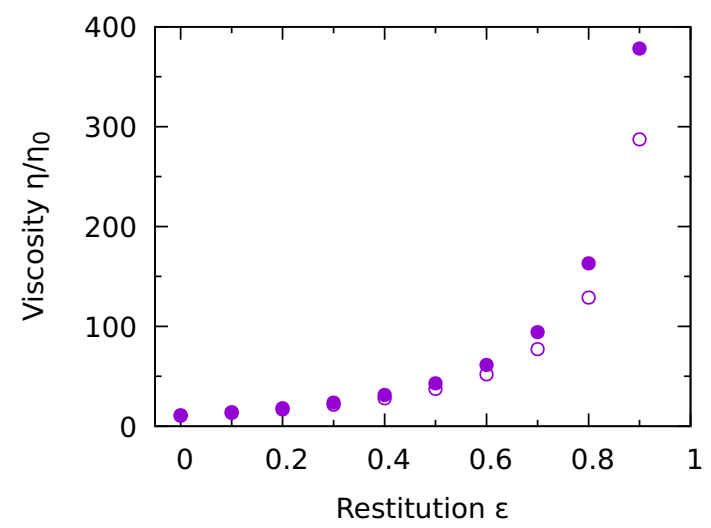

Figure 1. Viscosity $\eta$ relative to its low density limit $\eta_{0}$ as a function of the coefficient of restitution $\epsilon$ for packing fraction $\varphi=0.51$ and stiffness $\Gamma=1000$ (open), and 1500 (filled).

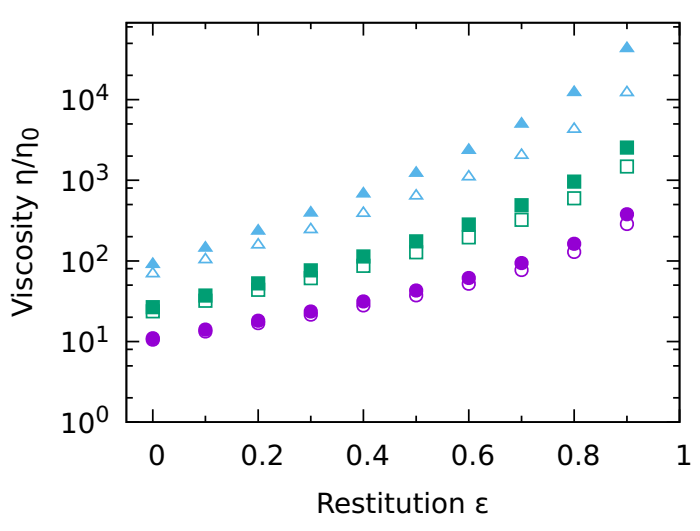

Figure 2. Viscosity $\eta$ relative to its low density limit $\eta_{0}$ as a function of the coefficient of restitution $\epsilon$ for packing fractions $\varphi=0.51$ (violet disks), 0.56 (green squares), and 0.61 (blue triangles) and stiffness $\Gamma=1000$ (open), and 1500 (filled).

$\eta_{0}$ valid for vanishing density. Also the stiffer particles have a higher viscosity. This increase is explained by the approach to the glass transition $\varphi_{c}(\Gamma)$ which is lower for stiffer particles [32, 34].

In general, the viscosity increases over several orders of magnitude upon increasing the density (cf. Fig. 2. Note that we span more than two orders of magnitude in viscosity by varying the inelasticity $\epsilon$ at the highest packing fraction considered.

\section{Nonlinear Rheology}

For strong shear such that shear heating dominates the fluidization, $\sigma \dot{\gamma} \gg n P_{D}$, we obtain Bagnold scaling where the fluidized bed no longer behaves like a Newtonian fluid, $\sigma=\eta \dot{\gamma}$, but $\sigma=B \dot{\gamma}^{2}$. This peculiar behavior of granular fluids has first been observed and described by Bagnold [35]. Kinetic predictions for the generalized (Bagnold) viscosity $B$ (which has dimensions of an inverse length) are sparse. 


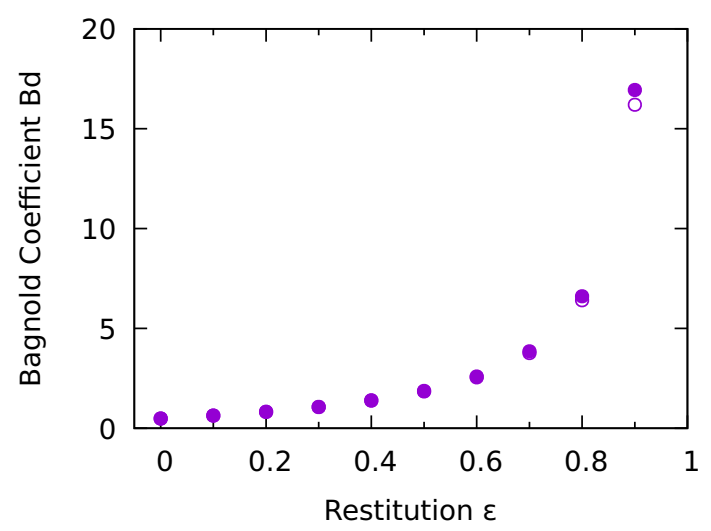

Figure 3. Bagnold coefficient $B \cdot d$ (where $d$ is the particle diameter) as a function of the coefficient of restitution $\epsilon$ for packing fraction $\varphi=0.51$ and two values of the stiffness $\Gamma=1000$ (open), and 1500 (filled).

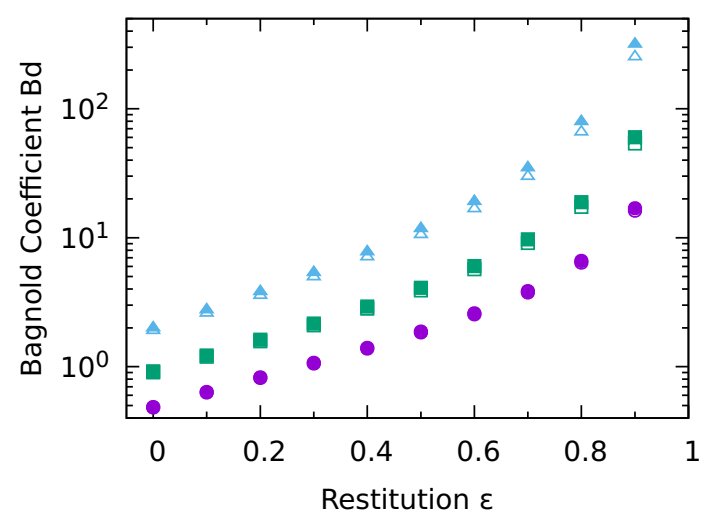

Figure 4. Bagnold coefficient $B \cdot d$ as a function of the coefficient of restitution $\epsilon$ for packing fractions $\varphi=0.51$ (violet disks), 0.56 (green squares), and 0.61 (blue triangles) and two values of the stiffness $\Gamma=1000$ (open), and 1500 (filled).

The ITT formalism sketched above yields an expression for the Bagnold coefficient [21] in terms of the coherent scattering function. Numerical evaluation of the resulting generalized Green-Kubo-integrals yields values for the Bagnold coefficient $B$.

We observe (cf. Fig. 3) a very weak dependence of $B$ on the stiffness $\Gamma$ but, just as for the viscosity, a strong increase with the coefficient of restitution $\epsilon$. As is expected also the Bagnold viscosity increases with increasing density (cf. Fig. 4)

\section{Discussion}

We presented first results from an granular ITT formalism for strongly sheared dense granular fluids. It yields kinetic theory expressions for the viscosity beyond the lowest order in density. These allowed us to calculate viscosities well above the Boltzmann viscosity $\eta_{0}$ and to determine the dependence on the inelasticity (quantified by the coefficient of restitution $\epsilon$ ) and on the parameter $\Gamma$ controlling the stiffness of the Hertzian potential. The general result is that the more elastic and the stiffer the particles are, the higher is the viscosity of the granular fluid.

We also presented predictions for the Bagnold coefficient $B$ which determines the non-Newtonian rheology of granular hard spheres at high shear rates. Like the Newtonian viscosity, it is a measure of the sluggishness of the fluid and thus increases towards the glass transition packing fraction of the quiescent fluid. We found that $B$ is rather insensitive to the stiffness $\Gamma$.

In the future we would expect our constitutive relations to be used in the modeling of dense granular at practically relevant shear rates. Kumaran [36] recently presented work in this direction.

\section{Acknowledgments}

We acknowledge continuing discussions with Annette Zippelius, Matthias Fuchs, and Fabian Frahsa and thank Marco Heinen for the structure factor code. W. T. K. acknowledges financial support by FOR1394.

\section{References}

[1] K. Hutter, N. Kirchner, Dynamic response of granular and porous materials under large and catastrophic deformations (Springer, 2013)

[2] M. Ausloos, R. Lambiotte, K. Trojan, Z. Koza, M. Pe et al., Physica A 357, 337 (2005)

[3] R.M. Iverson, Rev. Geophys. 35, 245 (1997)

[4] J.J. Lissauer, Annu. Rev. Astron. Astrophys. 31, 129 (1993)

[5] H.M. Jaeger, S.R. Nagel, Science 255, 1523 (1992)

[6] D. Kunii, O. Levenspiel, Fluidization engineering (Elsevier, 1991)

[7] D. Brunsden, Geomorphology 30, 13 (1999)

[8] C.S. Campbell, Powder Technology 162, 208 (2006)

[9] L.E. Silbert, D. Ertaş, G.S. Grest, T.C. Halsey, D. Levine, S.J. Plimpton, Phys. Rev. E 64, 051302 (2001)

[10] X. Cheng, J.B. Lechman, A. Fernandez-Barbero, G.S. Grest, H.M. Jaeger, G.S. Karczmar, M.E. Möbius, S.R. Nagel, Phys. Rev. Lett. 96, 038001 (2006)

[11] C.H. Rycroft, G.S. Grest, J.W. Landry, M.Z. Bazant, Phys. Rev. E 74, 021306 (2006)

[12] N.V. Brilliantov, T. Pöschel, Kinetic theory of granular gases (Oxford University Press, 2010)

[13] T. Pöschel, S. Luding, eds., Granular gases, Vol. 564 (Springer Science \& Business Media, 2001)

[14] V. Garzó, J.M. Montanero, Physica A 313, 336 (2002)

[15] M. Fuchs, M.E. Cates, Phys. Rev. Lett. 89, 248304 (2002)

[16] J.M. Brader, M.E. Cates, M. Fuchs, Phys. Rev. Lett. 101, 138301 (2008)

[17] M. Fuchs, M.E. Cates, J. Rheol. 53, 957 (2009)

[18] A.R. Abate, D.J. Durian, Phys. Rev. E 74, 031308 (2006) 
[19] W.T. Kranz, M. Sperl, A. Zippelius, Phys. Rev. Lett. 104, 225701 (2010)

[20] W.T. Kranz, M. Sperl, A. Zippelius, Phys. Rev. E 87, 022207 (2013)

[21] W.T. Kranz, F. Frahsa, A. Zippelius, M. Fuchs, M. Sperl, in preparation

[22] H. Hayakawa, M. Otsuki, Prog. Theor. Phys. 119, 381 (2008)

[23] K. Suzuki, H. Hayakawa, Phys. Rev. Lett. 115, 098001 (2015)

[24] J.C. Pàmies, A. Cacciuto, D. Frenkel, J. Chem. Phys. 131, 044514 (2009)

[25] L.D. Landau, E.M. Lifshitz, Theory of elasticity (Pergamon Press Oxford, 1986)

[26] R.P. Ojha, P.A. Lemieux, P.K. Dixon, A.J. Liu, D.J. Durian, Nature 427, 521 (2004)

[27] M. Schröter, D.I. Goldman, H.L. Swinney, Phys. Rev. E 71, 030301 (2005)
[28] G.P. Morriss, D.J. Evans, Statistical Mechanics of Nonequilbrium Liquids (ANU Press, 2007)

[29] S. Chapman, T.G. Cowling, The mathematical theory of non-uniform gases: an account of the kinetic theory of viscosity, thermal conduction and diffusion in gases (Cambridge University Press, 1970)

[30] W. Götze, Complex dynamics of glass-forming liquids: A mode-coupling theory, Vol. 143 (OUP Oxford, 2008)

[31] G.A. Martynov, G.N. Sarkisov, Mol. Phys. 49, 1495 (1983)

[32] M. Heinen, M. Sperl, in preparation

[33] S. Torquato, F.H. Stillinger, Rev. Mod. Phys. 82, 2633 (2010)

[34] L. Berthier, A.J. Moreno, G. Szamel, Phys. Rev. E 82, 060501 (2010)

[35] R.A. Bagnold, Proc. Royal Soc. A 225, 49 (1954)

[36] V. Kumaran, J. Fluid Mech. 756, 555 (2014) 